Article

\title{
Psychophysiological Measures of Reactance to Persuasive Messages Advocating Limited Meat Consumption
}

\author{
Hanne Spelt ${ }^{1,2, * \mathbb{D}}$, Elisabeth Kersten-van Dijk ${ }^{2}$, Jaap Ham ${ }^{2}$, Joyce Westerink ${ }^{1,2} \mathbb{D}$ and \\ Wijnand IJsselsteijn ${ }^{2}$ \\ 1 Philips Research, 5656 Eindhoven, The Netherlands; joyce.westerink@philips.com or \\ j.h.d.m.westerink@tue.nl \\ 2 Department of Industrial Engineering and Innovation Sciences, Eindhoven University of Technology, \\ 5612 AZ Eindhoven, The Netherlands; e.t.v.dijk@tue.nl (E.K.-v.D.); j.r.c.ham@tue.nl (J.H.); \\ w.a.ijsselsteijn@tue.nl (W.I.) \\ * Correspondence: hanne.spelt@philips.com or h.a.a.spelt@tue.nl; Tel.: +31-6-15134665
}

Received: 20 August 2019; Accepted: 16 October 2019; Published: 17 October 2019

\begin{abstract}
Persuasive interventions can lose their effectiveness when a person becomes reactant to the persuasive messages - a state identified by feelings of anger and perceived threat to freedom. A person will strive to reestablish their threatened freedom, which is characterized by motivational arousal. Research suggests that the motivational state of psychological reactance can be observed in physiology. Therefore, the assessment of physiological reactions might help to identify reactance to persuasive messages and, thereby, could be an objective approach to personalize persuasive technologies. The current study investigates peripheral psychophysiological reactivity in response to persuasive messages. To manipulate the strength of the reactant response either high- or low-controlling language messages were presented to discourage meat consumption. The high-controlling language condition indeed evoked more psychological reactance, and sympathetic arousal did increase during persuasive messaging in heart rate and heart rate variability, although no clear relationship between physiological reactivity and self-reported psychological reactance was found. However, the evaluation of multiple linear models revealed that variance in self-reported psychological reactance was best explained by initial intentions in combination with cardiovascular reactivity. To conclude, considering physiological reactivity in addition to motivational state can benefit our understanding of psychological reactance.
\end{abstract}

Keywords: psychophysiology; cardiovascular arousal; electrodermal arousal; persuasion profiling; psychological reactance

\section{Introduction}

Persuasive technologies can help people to change their behavior to become healthier or more pro-environmental by presenting persuasive information or indicating opportunities for change. However, a persuasive message may also evoke psychological reactance. In that case the user is motivated to reject the advocacy, thereby limiting the desired impact of the persuasive technology on behavior [1]. The motivational state and the negatively valenced emotions associated with psychological reactance are likely-as any emotions-to be reflected in psychophysiological signals [2-4]. Physiological reactions might then be used to detect whether a persuasive message is evoking resistance [5]. As such, physiology could be an objective measure of persuasion effectiveness. Physiology-based selection of persuasive content would enable unobtrusive personalization of persuasive technologies, minimizing the occurrence of reactance. In theory, such affective-loop systems [6] facilitate user-specific tailoring and help to improve long-term behavior change interventions 
within and across individuals, contexts and time. Affective-loop systems could then contribute to the field of personalized persuasive technologies. This study investigates the physiological patterns in the cardiovascular and electrodermal systems that occur when people respond to persuasive messages that can give rise to psychological reactance.

\subsection{Psychological Reactance Is Situation Specific}

Persuasive messages aim at convincing people to change their attitudes, intentions and behaviors [7], but can also be perceived as a threat to or restriction of certain freedoms [7,8], e.g., due to the use of controlling or forceful language [5]. In that case, people experience psychological reactance in which a motivation is aroused to reject the advocacy and reestablish their threatened freedom [3]. Psychological reactance is a reactive phenomenon-it occurs when a person responds to a situation containing a specific threat to a specific freedom and is best described as a mix of negative cognitions and emotions towards this threat $[1,7]$. The negative emotions and cognitions aroused depend on these situational characteristics [7]. To overcome feelings of reactance, a person may engage in freedom restoration behaviors with a state of motivational arousal [1,3,9].

Differences between people and/or the strength of threats can influence the level of the reactant response. Individual differences arise from the perceived importance of the freedoms that are threatened [9]. The beliefs that shape perceived importance rely on underlying motivations, such as social norms [1] or intentions [5,10]. A second determinant for the magnitude of reactance is the nature and strength of the threat, which can depend on the content but also on the formulation of the message $[8,11,12]$. Generally, high controlling language (HCL) is more likely to arouse reactance than low controlling language (LCL): HCL has a powerful and directive nature due to the use of many imperatives. It tends to be short, clear and efficient [12]. In LCL, the intentions of the sender are more ambiguous. LCL emphasizes self-initiation and choice. Consequently, it is perceived to be more polite and less forceful [12]. Usage of HCL increases the probability that the recipient perceives the messages as a threat, will reject the message, and experiences psychological reactance [12].

\subsection{Measuring the Psychophysiology of Reactant Responses}

Earlier research indicates that it is difficult to measure the presence and intensity of reactant responses and its effects and on people's experiences [1,8]. Several surveys have been developed for this purpose $[1,5,8]$, but their validity is an ongoing debate $[5,8]$. Most surveys measure trait characteristics of reactance, while reactance is a situational response [1]. Therefore, researchers have proposed physiological measures as an additional measure of reactance accounting for direct affective responses $[1,5]$. Physiological arousal can give information about the mental state of a person, and can thereby function as an implicit measure of the mind $[13,14]$. Therefore, analyzing psychophysiological responses might yield essential additional insights into psychological reactance.

For our argumentation, it is important to realize that the motivational state of reactance has energizing properties, which can be reflected in the physiological system [1,5]. That is, cortical responses reflecting the negative emotions and cognitions specific to reactant responses can influence peripheral physiology as well [2]. Peripheral physiology is influenced by the sympathetic, 'fight-or-flight', and parasympathetic, 'rest-and-digest', branches of the nervous system. These influences are measurable, among others, using features of the cardiovascular and electrodermal system [15,16]. Thus, the negative emotions and cognitions that arise in reactant responses might reflect in cardiovascular and electrodermal arousal $[17,18]$. To draw psychophysiological inferences from cardiovascular and electrodermal arousal we have to review the function of both systems.

The cardiovascular system is responsible for blood circulation and, thereby, transportation of blood cells, oxygen, nutrients, waste, and hormones through the body [16]. Easily measurable features of the cardiovascular system are heart rate (HR), i.e., the number of heart beats per minute, and heart rate variability (HRV), i.e., the variability between those beats resulting from the interplay between the sympathetic and parasympathetic nervous system [19]. Sympathetic influences increase HR and 
decrease HRV, and can hint at high arousal emotions, such as fear or joy, and increased cognitive demands. HR decreases and HRV increases under parasympathetic control, often indicating resting states or passive emotions [2,20]. Electrodermal activity, on the other hand, comprises sweat gland activity and is under only sympathetic control. Electrodermal arousal can increase in actionable emotional experiences and cognitive demands, such as decision-making. Measurable features of electrodermal arousal are the tonic component, namely skin conductance level (SCL), and the number of rapid phasic responses per minute, called skin conductance responses (SCRs) [21].

Results from several studies indeed indicated that psychological reactance can be seen as a state with motivational, emotional and cognitive components $[3,5,8,22,23]$, such as anger, which is both motivational and affective [5], and negative cognitions [8]. Earlier research has shown that reactant responses are indeed associated with heightened sympathetic arousal as reflected in elevated epinephrine and norepinephrine neurotransmitter levels [1]. Only a few studies have linked peripheral physiological arousal with psychological reactance $[3,5,23]$. These studies analyzed reactance in specific contexts and only for heart rate and skin conductance levels. Other features of cardiovascular and electrodermal arousal such as heart rate variability or skin conductance responses are even more sensitive to sympathetic and parasympathetic changes [16], and, thereby, might reveal further insights in psychological reactance. Evoking reactance in other contexts can also produce extra insights, as situational aspects might determine psychophysiological arousal during reactance, for example, the topic, the severity of the threat, the perceived importance of the freedom, or specific determinants of freedom.

\subsection{Study Aim and Hypotheses}

This study investigates whether psychological reactance is reflected in peripheral physiology. Additionally, we want to gain more insight in what predicts psychological reactance, especially the potential role physiological arousal. We build on previous work on reactance to persuasion [7] by using a similar experiment set-up with persuasive content differing in high or low controlling language and self-report measures of reactance $[7,24]$, but now also measure cardiovascular and electrodermal activity. Doing so, previously researched $[3,23]$ and new features of the cardiovascular and electrodermal system will be analyzed in relation to psychological reactance. To induce reactant responses, we will use the issue of meat eating, because earlier research suggested people consume meat as a way to express their identity [25] and that reactance occurs more easily for high involvement issues [11]. Based on earlier research we expect that 1) the motivational arousal and negative feelings in reactance increase sympathetic arousal, as reflected by HR acceleration, decreased HRV, elevated SCL and more SCRs compared to rest state, 2) that this increased arousal positively correlates with self-report measures of reactance, and 3) this psychophysiological relation adds insights to current means of predicting relationship explains part of the reactant responses.

\section{Method}

\subsection{Participants}

We recruited participants using the University database, which contains mainly students and a few adults. Fifty-nine people with a relatively high meat consumption ( $>5$ times per week) and without (a history of) cardiovascular diseases participated in this study. Sufficient English language skills and willingness to sign the informed consent were required. Participants received 10 euros or student credits as compensation. Participants were divided into two manipulation groups (group 1: $\mathrm{N}=31$ (19 women), $\mathrm{M}_{\mathrm{age}}=23.3, \mathrm{SD}_{\mathrm{age}}=5.5$, and group 2: $\mathrm{N}=28$ (16 women), $\mathrm{M}_{\mathrm{age}}=24.5, \mathrm{SD}_{\text {age }}=6.7$. The internal ethical board at the Eindhoven University of Technology reviewed and approved the study. 


\subsection{Manipulation}

This study had a between-subject repeated-measures design in which participants watched a persuasive video advocating limited meat consumption in either high controlling (HCL) or low controlling language (LCL) for group 1 and 2 respectively. Based on previous research $[7,24]$, the video consisted of a threat-to-health/environment component (202 words) and a recommendation for action (Table 1, HCL: 226 words, LCL: 224 words). The first part emphasized the negative consequences of behavior, whereas the second part regulated the strength of the threat. Both videos had comparable content but differed in framing. Besides using more imperatives, the HCL video pressed participants to do a certain action, i.e., become vegetarian, whereas the LCL video emphasized the choice for action. Especially for the group that saw the HCL video, the intervention tried to evoke psychological reactance.

Table 1. A subset of sentences from the manipulation video advocating limited meat consumption.

\begin{tabular}{|c|c|}
\hline Segment & Example Sentences \\
\hline $\begin{array}{l}\text { Threat-to-health/environment } \\
\text { (202 words, presented to both } \\
\text { groups) }\end{array}$ & 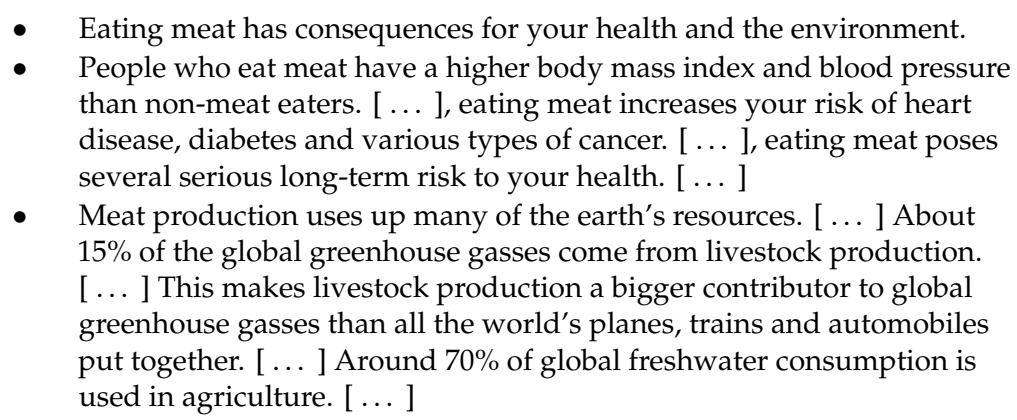 \\
\hline $\begin{array}{c}\text { Recommendation in HCL } \\
\text { (226 words, presented to group 1) }\end{array}$ & $\begin{array}{l}\text { - As any sensible person can see, there is really no choice when it comes to } \\
\text { consuming meat: you simply have to stop. }[\ldots] \\
\text { - The scientific evidence showing a link between cardiovascular risks and } \\
\text { meat consumption is so overwhelming that only a fool would possibly } \\
\text { argue with it. [... ] } \\
\text { - If you have been reducing your meat consumption, do it even more. [ ... } \\
\text { ] If you haven't been reducing your meat consumption, right now is the } \\
\text { time to start. Today. [ ... ] } \\
\text { - Set a goal for yourself to stop and commit to it. Stop eating meat. }\end{array}$ \\
\hline $\begin{array}{c}\text { Recommendation in LCL } \\
\text { (224 words, presented to group 2) }\end{array}$ & $\begin{array}{l}\text { - Most people agree that reducing your meat consumption is a good idea; } \\
\text { nevertheless, the choice to do so is completely up to you. [ . . . ] } \\
\text { You are the boss of your own body and you make the rules. What you } \\
\text { consume is your own decision. [ . . . ] } \\
\text { - If you have been reducing your meat consumption, we support your } \\
\text { decision. And if you haven't been reducing your meat consumption, we } \\
\text { support your decision. [ ... ] } \\
\text { - You are free to do as you want. }\end{array}$ \\
\hline
\end{tabular}

\subsection{Procedure}

One week before the laboratory session, the participant completed an online pre-survey assessing demographic information, meat consumption, and initial motivational state towards limited meat consumption. The participant was instructed to refrain from caffeinated drinks in the 2 hours preceding the laboratory session. Prior to the experiment segments, the participant received a short introduction, signed the informed consent and was attached to the physiological measurement equipment. Then, the participant was seated in front of a computer screen to start the experiment. The computer asked the participant to describe his/her favorite dish to increase awareness of their consumption freedom (Figure 1). A baseline measurement of physiology was conducted twice while the participant viewed 3- and 5-minute sea-life movies [26]. A 4.5-minute factual video with neutral information about 
the consequences of meat consumption on the environment and health followed the first baseline. The factual video ensured that all participants had similar topic-specific knowledge. After the second baseline, the persuasive messages were presented. Both the HCL and LCL messages had a total duration of 3 minutes. After the persuasive messages, participants had the opportunity to restore their freedom by filling out questions in the post-survey while reflecting on the video. In this post-survey, a reactance questionnaire as well as control questions were asked in addition to questions assessing motivational state towards limited meat consumption.

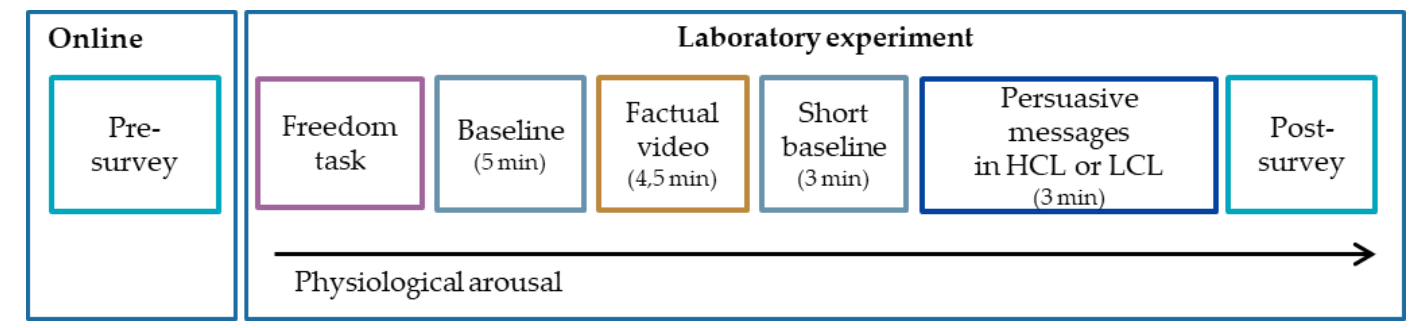

Figure 1. Experimental procedure during the laboratory experiment starting with a freedom exercise followed by a baseline measurement of physiology in rest, a factual movie about the consequences of meat consumption on health and environment, a second baseline, a persuasive message using high or low controlling language and a post-survey.

\subsection{Materials}

\subsubsection{Subjective Data}

Self-report measures included demographic information such as age, gender and educational level. The highest received degree determined educational level. Following the theory of planned behavior [10,20], participants' motivational state to limit meat consumption was asked using questions about attitude towards and intention to perform the advocated behavior, as well as subjective and injunctive norms. The five attitude items focused on the instrumental, e.g., worthless-valuable, and affective, e.g., good-bad, nature of limiting meat consumptions with scale end-points counterbalanced. Intention was assessed using three items on intentional effort. Subjective norms measured the perceived expectations of other people's behavior ( 3 items), whereas injunctive norms represented people's ideas of the participants own behavior ( 3 items). These items used a 7-point Likert scale ranging from 'strongly disagree' to 'strongly agree'.

In the post-survey, four items per scale concerning feelings of anger and perceived threat to freedom (PTTF) checked if participants were reactant towards the video. These questionnaires were answered on 5-point Likert scales ranging from 'completely disagree' to 'completely agree' (Table 2) [7]. In addition, a control survey consisted of one question on the newness of the information and three multiple-choice questions to test if participants paid attention to the video. The questions were presented in English. With exception of the control questions, all questionnaires in this study were validated in previous research and analyzed as instructed $[7,10]$ using various packages in $R$ studio [27-29].

Table 2. Items for both reactance scales.

\begin{tabular}{ccc}
\hline & Feelings of Anger & Perceived Threat to Freedom \\
\hline 1 & I was irritated & The video tried to make a decision for me \\
2 & I was angry & The video tried to manipulate me \\
3 & I was annoyed & The video tried to pressure me \\
4 & I was aggravated & The video threatened my freedom to choose \\
\hline
\end{tabular}




\subsubsection{Physiological Data}

We used a Mobi physiology-recording device with gel electrodes in Lead II placement for ECG measurement, and dry electrodes with Velcro straps on the fingertips for skin conductance measurement [21], sampling at $1029.5 \mathrm{~Hz}$. Physiological features were measured during the complete laboratory experiment. In the ECG signal, we calculated inter-beat intervals (IBIs) and verified them by manually checking the R-peaks. IBIs below $0.4 \mathrm{~s}$ or above $1.4 \mathrm{~s}$ were interpolated [30]. This procedure was not seldom needed. From the filtered IBI data, mean heart rate (HR), standard deviation from normal-to-normal peaks (SDNN), and root mean square of successive differences (RMSSD) for the middle three minutes of each experiment segment were calculated. Electrodermal activity (EDA) was down-sampled to $2 \mathrm{~Hz}$ and filtered with a $0.5 \mathrm{~Hz}$ low-pass Butterworth filter. From the filtered EDA signal, mean skin conductance level (SCL) and the number of skin conductance response peaks per second (SCRs) were calculated for the middle 3 minutes of each experiment segment [16]. The difference between the physiological values during the factual video, persuasive messages, or survey and those of the preceding baseline served as measure of physiological reactivity to each experiment segment, e.g., reactivity (messages) $=\operatorname{arousal}_{\text {(messages) }}-\operatorname{arousal}_{\text {(short baseline) }}$. Several $\mathrm{R}$ packages were used for the preprocessing of physiological data $[28,30,31]$.

\subsection{Analyses}

First, we verified if the two groups were statistically similar with respect to demographic information and motivational state using an independent t-test. As manipulation check, an independent t-test was applied to check whether HCL evoked more reactance than LCL. Additionally, a within-between MANOVA on attitudes and intentions checked whether the video was persuasive.

To answer hypothesis 1, a linear mixed model was applied for each physiological reactivity variable with experimental segment and manipulation condition as fixed and subject as random effects. In linear mixed models, fixed effects are variables constant over measurements, while random effects can vary per measurements. This approach enabled the analysis of physiological reactivity during different experiment segments, while accounting for individual differences and missing data [32].

To answer hypotheses 2 and 3, we evaluated the fit of multiple linear models with psychological reactance as dependent variable. To yield only one dependent variable, reactance was calculated by adding anger and PTTF scores. The best predictors of variance in psychological reactance were established by evaluating four models: (1) a null model, (2) a state model, (3) a message-reactivity model, and (4) a full model. The null model included no predictors. The state model evaluated all self-reported scores that determined initial motivational state to limit meat consumptions; attitude, intention, subjective and injunctive norms before the manipulation. The message-reactivity model evaluated HR, SDNN, RMSSD, SCL and SCRs reactivity to the persuasive messages-that is the rise in physiological arousal from the short baseline to the persuasive messages for each parameter. Lastly, the full model evaluated all physiological reactivity and initial motivational state variables. Only significant models were presented and only including those variables that improved predictive power of the model by explaining extra variance in reactance based on AIC weights [33]. Eventually, the fit of the four models was evaluated using Akaike Information Criterion [33]. This evaluation reveals which combination of predictor variables predicts variance in psychological reactance best. In these relational analyses, the effect of manipulation was not considered relevant. Analyses were carried out using several R Studio packages [27-29,34].

\section{Results}

The final data set contained subjective and physiological data of 56 participants. Three datasets had to be excluded due to incompleteness. Data for intention, descriptive norms, and PTTF were not normally distributed and transformation did not effectuate normal distribution, i.e., $\log , \log +1$, Tukey's Ladder of Powers, Cube root nor square root transformation. There were no significant differences 
between the demographic characteristics or initial motivational state of the two groups, e.g., attitudes, intention, subjective norms and descriptive norms.

As a persuasion check, a within-between MANOVA with Bonferroni-Holm correction was conducted to compare the main effects of condition and time, and the interaction effect between manipulation and time on attitudes and intentions towards limited meat consumption. Both the main and the interaction effects were not significant; attitudes and intentions did not change over the course of the experiment in either condition.

As a manipulation check, an independent sample one-tailed Mann-Whitney U t-test with Bonferroni-Holm correction revealed that the HCL condition indeed evoked significantly more anger and perceived threat to freedom than the LCL persuasive messages (Table 3).

Table 3. Descriptive statistics of both reactance scales and results of a one-tailed Mann-Whitney U test comparing the two conditions (high controlling language $(\mathrm{HCL})>$ low controlling language (LCL)).

\begin{tabular}{|c|c|c|c|c|c|c|c|}
\hline Condition & & & & & & & \\
\hline Scale & $\alpha$ & $\mathrm{m}$ & s.d. & $\mathrm{m}$ & s.d. & $\mathrm{U}$ & $p$ \\
\hline Anger & 0.91 & 3.45 & 1.35 & 2.84 & 1.12 & 280 & 0.034 \\
\hline PTTF & 0.92 & 4.20 & 1.43 & 2.70 & 1.45 & 164 & 0.002 \\
\hline
\end{tabular}

The results of multiple linear mixed models with experiment segment and manipulation condition as fixed effects and subject as random effect showed physiological reactivity differed significantly between experimental segments for HR, SDNN, and RMSSD (Table 4). On average, SDNN and RMSSD were 89 and $36 \mathrm{~ms}$ lower, and HR $1.30 \mathrm{bpm}$ higher during the persuasive messages compared to the short baseline. During the factual video, HR reactivity was $1.03 \mathrm{bpm}$ lower than during the persuasive messages, whereas SDNN reactivity was $54 \mathrm{~ms}$ higher (see also Figure 2). With the exception of number of skin conductance peaks, the difference in experiment segments explains between $32.7 \%$ and $44.9 \%$ of the variance in physiological reactivity based on the conditional $\mathrm{R}^{2}$, i.e., based on both fixed and random effects [34]. There was no significant effect of manipulation on physiological reactivity.

Table 4. Summary results of the mixed linear models for reactivity of Heart Rate (HR), Heart Rate Variability (standard deviation from normal-to-normal peaks (SDNN) and root mean square of successive differences (RMSSD)) and Electrodermal (skin conductance level (SCL) and skin conductance responses (SCRs)) per segment.

\begin{tabular}{|c|c|c|c|c|c|c|c|c|c|c|}
\hline \multirow[b]{2}{*}{ Predictors } & \multicolumn{2}{|c|}{ HR } & \multicolumn{2}{|c|}{ SDNN $\cdot 10^{2}$} & \multicolumn{2}{|c|}{ RMSSD $\cdot 10^{2}$} & \multicolumn{2}{|c|}{ SCL } & \multicolumn{2}{|c|}{ SCRs } \\
\hline & Est. ${ }^{1}$ & $p$ & Est. & $p$ & Est. & $p$ & Est. & $p$ & Est. & $p$ \\
\hline $\begin{array}{c}\text { Persuasive messages } \\
\text { (Intercept) }\end{array}$ & 1.30 & 0.001 & -0.89 & $<0.001$ & -0.36 & 0.040 & 0.06 & 0.294 & -4.56 & 0.190 \\
\hline Factual video & -1.03 & 0.018 & 0.54 & 0.038 & 0.04 & 0.829 & -0.06 & 0.317 & -0.32 & 0.948 \\
\hline \multirow[t]{2}{*}{ Survey } & -0.60 & 0.165 & 0.50 & 0.058 & 0.13 & 0.519 & 0.10 & 0.112 & 4.74 & 0.330 \\
\hline & \multicolumn{6}{|c|}{ Random Effects } & & & & \\
\hline$\sigma^{2}$ & \multicolumn{2}{|c|}{5.25} & \multicolumn{2}{|c|}{2.26} & \multicolumn{2}{|c|}{1.10} & \multicolumn{2}{|c|}{0.11} & \multicolumn{2}{|c|}{639.99} \\
\hline ICC & \multicolumn{2}{|c|}{0.43} & \multicolumn{2}{|c|}{0.37} & \multicolumn{2}{|c|}{0.33} & \multicolumn{2}{|c|}{0.31} & \multicolumn{2}{|c|}{0.03} \\
\hline Obs. & \multicolumn{2}{|c|}{171} & \multicolumn{2}{|c|}{171} & \multicolumn{2}{|c|}{171} & \multicolumn{2}{|c|}{164} & \multicolumn{2}{|c|}{165} \\
\hline $\mathrm{R}^{2} /$ Cond. $\mathrm{R}^{2}$ & \multicolumn{2}{|c|}{$0.025 / 0.449$} & \multicolumn{2}{|c|}{$0.010 / 0.376$} & \multicolumn{2}{|c|}{$0.001 / 0.336$} & \multicolumn{2}{|c|}{$0.028 / 0.327$} & \multicolumn{2}{|c|}{$0.008 / 0.040$} \\
\hline AIC & \multicolumn{2}{|c|}{847.072} & \multicolumn{2}{|c|}{692.645} & \multicolumn{2}{|c|}{563.667} & \multicolumn{2}{|c|}{163.913} & \multicolumn{2}{|c|}{1549.650} \\
\hline
\end{tabular}

${ }^{1}$ Est. = estimated difference in units of the physiological parameters, $\mathrm{p}=\mathrm{p}$-value (presented in bold if significant), $\sigma 2=$ subject variance, ICC $=$ intra-class correlation coefficient, $R^{2}=$ Marginal $r$-squared statistics, Cond. $\mathrm{R}^{2}=$ conditional $r$-squared statistics, Obs. = number of observations, AIC = Akaike Information Criterion 


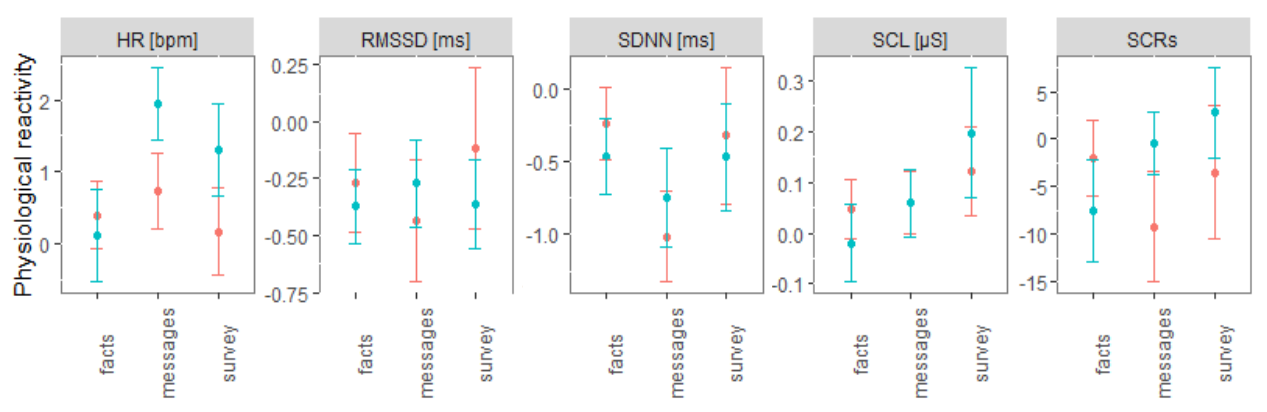

Figure 2. Average physiological reactivity per segment for each experimental group with error bars representing standard errors of the mean. Red $=$ group that received HCL, blue = group that received LCL.

Results of various linear models with reactance as dependent variable and physiological reactivity and/or initial motivational state as predictor variables for the main analysis are presented in Table 5 . Out of the four possible models (Section 2.5) only three models had a significant fit; the null, state, and full model. The null model includes no predictors and the significant intercept reveals that on average the participants experienced reactance. Results from the state model reveal that from all initial motivational state factors, e.g., attitude, injunctive and subjective norms, only intention to limit meat consumption explains variance in reactance; reported reactance drops 0.54 on a 1-14 point scale with each unit rise of initial intention. We did not find a significant fit for the message-reactivity model, suggesting a minor role of the physiological reactivity variables in explaining variance in reactance. However, the full model was significant, not only including a relationship between reactance and initial motivational state, but also with physiological reactivity. Results of the full model reveal that on average people report 8.73 experienced reactance on a 1-14 scale. Higher initial intentions to limit meat consumption lower the reported reactance by 0.58 per step on the intention scale. Although the physiological reactivity variables were non-significant on their own, they did yield a model with higher predictive power when combined with intention than the state model. Physiological reactivity to the persuasive messages also lowers reactance by 0.23 and 0.52 for each bpm rise in HR and millisecond rise in RMSSD, respectively. In comparison to the null and state models, the full model has the best fit based upon the lowest AIC. The full model explains around $20.1 \%$ of variance in self-reported reactance in our sample based on $R^{2}$.

Table 5. Results of three linear models explaining variance in reactance.

\begin{tabular}{|c|c|c|c|c|c|c|}
\hline \multirow[b]{2}{*}{ Predictors } & \multicolumn{2}{|c|}{ Null Model } & \multicolumn{2}{|c|}{ State Model } & \multicolumn{2}{|c|}{ Full Model } \\
\hline & Est. $^{1}$ & $p$ & Est. & $p$ & Est. & $P$ \\
\hline (Intercept) & 6.63 & $<0.001$ & 8.47 & $<0.001$ & 8.73 & $<0.001$ \\
\hline Intention to limit meat consumption & & & -0.54 & 0.012 & -0.58 & 0.006 \\
\hline HR reactivity to persuasive messages & & & & & -0.23 & 0.056 \\
\hline RMSSD reactivity to persuasive messages & & & & & -0.52 & 0.055 \\
\hline Obs. & \multicolumn{2}{|c|}{56} & \multicolumn{2}{|c|}{56} & \multicolumn{2}{|c|}{56} \\
\hline $\mathrm{R}^{2} /$ Adj. $\mathrm{R}^{2}$ & \multirow{2}{*}{\multicolumn{2}{|c|}{$0.000 / 0.000$}} & \multicolumn{2}{|c|}{$0.112 / 0.095$} & \multicolumn{2}{|c|}{$0.201 / 0.155$} \\
\hline AIC & & 262.250 & \multicolumn{2}{|c|}{257.627} & \multicolumn{2}{|c|}{255.693} \\
\hline
\end{tabular}

${ }^{1}$ Est. = estimated difference in units of the physiological parameters, $p=p$-value (presented in bold if significant), $\mathrm{R}^{2}=$ r-squared statistics, Adj. $\mathrm{R}^{2}=\mathrm{r}$-squared statistics adjusted for number of parameters, Obs. = number of observations, $\mathrm{AIC}=$ Akaike Information Criterion.

\section{Discussion}

Use of psychophysiological reactions could be an objective approach to personalize persuasive interventions, e.g., trying to avoid reactance. Therefore, we studied psychophysiological reactance to persuasive messages that used high controlling (HCL) or low controlling language (LCL). The messages 
tried to persuade people with a high meat consumption, e.g., $>5$ days per week, towards a more vegetarian diet. Psychological reactance to these messages was assessed with self-reported feelings of anger and perceived threat to freedom (PTTF). A factual video preceding the messages ensured that all participants had akin topic-specific knowledge. Motivations to limit meat consumption were measured one week before and immediately after the experiment. Physiological reactivity was measured during the factual video, the persuasive messages, and the closing survey, using features of the cardiovascular and electrodermal system.

We found that neither the HCL nor LCL messages persuaded participants to (further) limit their meat consumption, as indicated by equal attitude and intention levels before and after the experiment. While for the HCL condition this finding was in line with our expectations, we expected the LCL messages to increase motivations to limit meat consumption. As both groups report some level of reactance (at least 2.7 PTTF on a 7-point scale), this might have limited the persuasiveness of the messages. Importantly, participants were more reactant in the HCL condition by experiencing higher feelings of anger and greater perceived threat to freedom compared to participants that received the LCL condition. Therefore, we can assume that, despite the lack of attitude change, our manipulation was successful.

During the persuasive messages, participants had heightened sympathetic physiological arousal as indicated by cardiovascular arousal compared to activity during rest state, i.e., the short baseline. On the other hand, during the factual video, we found decreased heart rate and increased heart rate variability. Because the action performed by the participant was similar during the factual video and the persuasive messages, namely watching an informative video, this finding cannot be attributed to a difference in general information processing or attention. In addition, both the factual video and the persuasive messages were concerned with the context of meat consumption. Generally heart rate decelerates with increased attention [16], whereas during our persuasive messages the opposite occurs. This finding, therefore, seems to suggest that elevated cardiovascular reactivity is indeed caused by the content of the persuasive messages. We did not find a different effect of the HCL and LCL framing on cardiovascular or electrodermal reactivity. One reason for the lack of this finding could be that the manipulation conditions were not distinct enough in their psychological effects; both conditions were not persuasive and both evoked some level of reactance.

Further analyses reveal a relationship between psychological reactance and initial motivations to limit meat consumption; people with higher intention to limit their meat consumption experienced lower reactance. This finding is not surprising. As the intentions of these people were in line with the advocated appeal, the messages were probably less threatening to them and, thus, evoked lower levels of reactance. Interestingly, adding cardiovascular measures significantly improved this explanatory model. Both an increase in HR and SDNN reactivity appear to lower the reported reactance in this study. These findings are somewhat surprising as increased HR indicates arousal, whereas increased SDNN indicates relaxation. As this is contradictory, future research is needed to replicate this cardiovascular relation. Despite this ambiguity, the combination of initial intention with cardiovascular measures did explain almost twice as much variance in reactance than initial intentions alone, i.e., $20.1 \%$ versus $11.2 \%$ as indicated by the $\mathrm{R}^{2}$ in Table 5 . This underlines our idea that psychological reactance might have a psychophysiological nature. It surely invites the combination of subjective self-report with objective physiological measures in future reactance research.

This study has an explorative nature and, thereby, comes with limitations. Since the study was limited to the context of meat consumption and concerns only people that have a high meat consumption patterns ( $>5$ days per week), the findings cannot be generalized. Eating animals is seen as a moral dilemma between the aversion to animal suffering and the desire to eat meat [25]. The moralization of vegetarianism is driven by strong affective responses, such as disgust and guilt [35]. Additionally, the formation of these beliefs depend on other attributes, i.e., experiences, characteristics, objects, than health behaviors. [36]. Thus, the psychology of morality is wired differently than health beliefs. Therefore, it could be that similarly framed persuasive messages concerning other contexts 
produce different or no physiological markers. Further work is required to establish if this relation also holds in other contexts, e.g., climate change, energy saving.

Another limitation might come from the manipulation not being strong enough, explaining the similar effects of both conditions. Although the spread in reactance scores enabled correlational analyses, it could be that too little people experienced high enough levels of reactance to evoke physiological reactivity. Previous research [7] reported anger and PTTF scores between 0.45-1.44 and $2.31-3.11$ on a $0-4$ scale, while we found an average anger score of 3.45 and PTTF score of 4.20 on a 1-7 scale in the HCL condition. The scores are relatively high, but not extreme. This could be one reason for not finding a stronger psychophysiological relationship in reactance. Future research should find out whether higher levels of reactance do reflect in physiology or whether such a robust relationship does not exist at all.

Lastly, personality traits were not considered in this study, while they might have explained some of our results. As mentioned in the introduction, reactant responses are determined by the perceived importance of the freedoms that are threatened [9]. These perceptions may differ based on personality traits. These traits can therefore mediate the reactance response, but they can also influence the physiological response. For example, trait characteristics such as approach-avoidance motivation are associated with other nervous system activity patterns [37], novelty seeking correlates negatively with low frequency HRV and LF/HF ratio [38] and cardiovascular arousal relates to neuroticism and agreeableness [39]. As our main finding indicates that HRV parameters explain variability in self-reported reactance, personality traits should also be considered in future research.

Despite its limitations, the study adds to our understanding of persuasive messages and their effects on physiology. Future research should try to replicate and extend these findings to different contexts, types of communication, and people. If an evident physiological marker for psychological reactance is found, it could have considerable implications for personalized persuasive technologies, i.e., indicating which messages are (not) effective for the user. It could set up the use of built-in affective loops regulated by physiological, affective, and behavioral interactions in human-technology interaction. Thereby, it would enable physiology-based tailoring as a personalization technique for persuasive technology.

\section{Conclusions}

We did not find clear psychophysiological responses related to reactance. Nevertheless, the results do encourage further research because the present findings indicate more cardiovascular arousal during persuasive messages-although most likely not linked to reactance or attitude change. Further research should not only consider the strategy to evoke reactance but also types of freedom and underlying psychological processes that are targeted.

Author Contributions: conceptualization, E.K.-v.D., J.W., W.I., J.H., H.S.; data curation, H.S.; formal analysis, H.S.; funding acquisition, J.W.; investigation, H.S.; methodology, E.K.-v.D, H.S., J.W.; project administration, E.K.-v.D., H.S.; software, H.S.; supervision, E.K.-v.D., J.W.; validation, H.S.; visualization, H.S.; writing—original draft, H.S.; writing-review and editing, J.W., J.H., H.S.

Funding: This research was funded by the H2020 INHERIT project, grant number 667364 .

Acknowledgments: We thank all volunteers and the Human-Technology Interaction Group at the Eindhoven University of Technology. Special thanks to Shutong Liu, Tim van den Driesschen and Anisa Fardhani Prasetyaningtyas.

Conflicts of Interest: The authors declare no conflict of interest. The funders had no role in the design of the study; in the collection, analyses, or interpretation of data; in the writing of the manuscript, or in the decision to publish the results.

\section{References}

1. Miron, A.M.; Brehm, J.W. Reactance Theory-40 Years Later. Z. Sozialpsychol. 2006, 37, 9-18. [CrossRef] 
2. Kreibig, S.D. Autonomic nervous system activity in emotion: A review. Biol. Psychol. 2010, 84, $394-421$. [CrossRef] [PubMed]

3. Sittenthaler, S.; Steindl, C.; Jonas, E. Legitimate vs. illegitimate restrictions-A motivational and physiological approach investigating reactance processes. Front. Psychol. 2015, 6, 1-11. [CrossRef] [PubMed]

4. Picard, R.W.; Vyzas, E.; Healey, J. Toward machine emotional intelligence: Analysis of affective physiological state. IEEE Trans. Pattern Anal. Mach. Intell. 2001, 23, 1175-1191. [CrossRef]

5. Steindl, C.; Jonas, E.; Sittenthaler, S.; Traut-Mattausch, E.; Greenberg, J. Understanding psychological reactance: New developments and findings. Z. Psychol. 2015, 223, 205-214. [CrossRef] [PubMed]

6. Fairclough, S.H. Fundamentals of physiological computing. Interact. Comput. 2009, 21, 133-145. [CrossRef]

7. Dillard, J.P.; Shen, L. On the nature of reactance and its role in persuasive health communication. Commun. Monogr. 2005, 72, 144-168. [CrossRef]

8. Rains, S.A. The nature of psychological reactance revisited: A meta-analytic review. Hum. Commun. Res. 2013, 39, 47-73. [CrossRef]

9. Brehm, J.W. A Theory of Psychological Reactance; Academic Press: Oxford, UK, 1966.

10. Ajzen, I. Constructing a TPB Questionnaire: Conceptual and Methodological Considerations. Available online: people.umass.edu/aizen/pdf/tpb.measurement.pdf (accessed on 22 August 2018).

11. Ghazali, A.; Ham, J.; Barakova, E.; Markopoulos, P. The influence of social cues in persuasive social robots on psychological reactance and compliance. Comput. Human Behav. 2018, 87, 58-65. [CrossRef]

12. Miller, C.H.; Lane, L.T.; Deatrick, L.M.; Young, A.M.; Potts, K.A. Psychological reactance and promotional health messages: The effects of controlling language, lexical concreteness, and the restoration of freedom. Hum. Commun. Res. 2007, 33, 219-240. [CrossRef]

13. Picard, R.W. Affective Computing Research; MIT Media Laboratory: Cambridge, MA, USA, 1995.

14. Cacioppo, J.T.; Berntson, G.G.; Larsen, J.T.; Poehlmann, K.M.; Ito, T.A. The psychophysiology of emotion. In Handbook of Emotions; Lewis, M., Haviland-Jones, J.M., Feldman Barrett, L., Eds.; Guilford Publications: New York, NY, USA, 2000; Volume 2, ISBN 978-1593856502.

15. Jänig, W. The autonomic nervous system and its coordination by the brain. In Handbook of Affective Sciences; Davidson, R.J., Scherer, K.R., Goldsmith, H.H., Eds.; Oxford University Press: Oxford, UK, 2003; pp. $135-186$.

16. Cacioppo, J.T.; Tassinary, L.G.; Berntson, G.G. The Handbook of Psychophysiology; Cacioppo, J.T., Tassinary, L.G., Berntson, G.G., Eds.; Cambridge University Press: New York, NY, USA, 2007; Volume 44, ISBN 9780521844710.

17. Shoemaker, J.K.; Norton, K.N.; Baker, J.; Luchyshyn, T.A. Forebrain organization for autonomic cardiovascular control. Auton. Neurosci. Basic Clin. 2015, 188, 5-9. [CrossRef] [PubMed]

18. Thayer, J.F.; Åhs, F.; Fredrikson, M.; Sollers, J.J.; Wager, T.D. A meta-analysis of heart rate variability and neuroimaging studies: Implications for heart rate variability as a marker of stress and health. Neurosci. Biobehav. Rev. 2012, 36, 747-756. [CrossRef] [PubMed]

19. Camm, A.J.; Malik, M.; Bigger, J.T.; Breithardt, G.; Cerutti, S.; Cohen, R.J.; Coumel, P.; Fallen, E.L.; Kennedy, H.L.; Kleiger, R.E.; et al. Heart rate variability: Standards of measurement, physiological interpretation and clinical use. Circulation 1996, 1043-1065.

20. Thayer, J.F.; Lane, R.D. Claude Bernard and the heart-brain connection: Further elaboration of a model of neurovisceral integration. Neurosci. Biobehav. Rev. 2009, 33, 81-88. [CrossRef]

21. Boucsein, W. Electrodermal Activity, 2nd ed.; Springer Science+Business Media: New York, NY, USA, 2012; ISBN 9781461411253.

22. Jonas, E.; Graupmann, V.; Kayser, D.N.; Zanna, M.; Traut-Mattausch, E.; Frey, D. Culture, self, and the emergence of reactance: Is there a "universal" freedom? J. Exp. Soc. Psychol. 2009, 45, 1068-1080. [CrossRef]

23. Sittenthaler, S.; Jonas, E.; Traut-Mattausch, E. Explaining self and vicarious reactance. Personal. Soc. Psychol. Bull. 2016, 42, 458-470. [CrossRef]

24. Rogers, R.W. Cognitive and psychological processes in fear appeals and attitude change: A revised theory of protection motivation. In Social Psychophysiology: A Source Book; Cacioppo, J.T., Shapiro, D., Eds.; Guilford Press: New York, NY, USA, 1983; pp. 153-176.

25. Loughnan, S.; Bastian, B.; Haslam, N. The psychology of eating animals. Curr. Dir. Psychol. Sci. 2014, 23, 104-108. [CrossRef]

26. Overbeek, T.J.M.; van Boxtel, A.; Westerink, J.H.D.M. Respiratory sinus arrhythmia responses to induced emotional states: Effects of RSA indices, emotion induction method, age, and sex. Biol. Psychol. 2012, 91, 128-141. [CrossRef] 
27. RStudio: Integrated Development Environment for R 2016, version 3.5.2; Eggshell Igloo, Rstudio Team: Boston, MA, USA, 2016.

28. Wickham, H. Tidyverse: Easily Install and Load the "Tidyverse"; RStudio: Boston, MA, USA, 2017.

29. Revelle, W. Psych: Procedures for Psychological, Psychometric, and Personality Research; Northwestern University: Evanston, IL, USA, 2017.

30. Zeileis, A.; Grothendieck, G.; Ryan, J.A.; Ulrich, J.M.; Andrews, F. Zoo: S3 Infrastructure for Regular and Irregular Time Series (Z's Ordered Observations); RStudio: Boston, MA, USA, 2019.

31. Carezia, A.; Dupuis, P.; Eaton, J.W.; Habel, K.; Hornik, K.; Krey, S.; Lash, B.; Leisch, F.; Smith, J.O.; Stewart, D.; et al. Signal Processing 2015; RStudio: Boston, MA, USA, 2015.

32. Venables, W.N.; Ripley, B.D. Modern Applied Statistics with S, 4th ed.; Chambers, J., Eddy, W., Härdle, W., Sheather, S., Tierney, L., Eds.; Springer Science+Business Media: New York, NY, USA, 2003; ISBN 978-1-4419-3008-8.

33. Wagenmakers, E.; Farrell, S. AIC model selection using Akaike weights. Psychon. Bull. Rev. 2004, 11, $192-196$. [CrossRef]

34. Bates, D.; Mächler, M.; Bolker, B.; Walker, S. Fitting linear mixed-effects models using lme4. J. Stat. Softw. 2015, 67, 1-48. [CrossRef]

35. Stoess, C.; Rozin, P.; Markwith, M. Moralization and becoming a vegetarian: The transformation of preferences into values and the recruitment of disgust. Psychol. Sci. 1997, 8, 67-73.

36. Ajzen, I. The theory of planned behavior. Organ. Behav. Hum. Decis. Process. 1991, 50, 179-211. [CrossRef]

37. Balconi, M.; Falbo, L.; Brambilla, E. BIS/BAS responses to emotional cues: Self report, autonomic measure and alpha band modulation. Pers. Individ. Dif. 2009, 47, 858-863. [CrossRef]

38. Takahashi, T.; Murata, T.; Hamada, T.; Omori, M.; Kosaka, H.; Kikuchi, M.; Yoshida, H.; Wada, Y. Changes in EEG and autonomic nervous activity during meditation and their association with personality traits. Int. J. Psychophysiol. 2005, 55, 199-207. [CrossRef] [PubMed]

39. Koelsch, S.; Enge, J.; Jentschke, S. Cardiac signatures of personality. PLoS ONE 2012, 7, e31441. [CrossRef] [PubMed] 